\title{
Chemical evolution of the Milky Way: the origin of phosphorus (Research Note)
}

\author{
G. Cescutti ${ }^{1}$, F. Matteucci ${ }^{2,3}$, E.Caffau ${ }^{4,5}$, and P. François ${ }^{5,6}$ \\ 1 Leibniz-Institut für Astrophysik Potsdam (AIP), An der Sternwarte 16, 14482 Potsdam, Germany \\ ${ }^{2}$ Laboratoire d'Astrophysique, École Polytechnique Fédérale de Lausanne (EPFL), Observatoire de Sauverny, 1290 Versoix, \\ Switzerland \\ e-mail: cescutti@aip.de \\ 3 Dipartimento di Fisica, Sezione di Astronomia, Università di Trieste, via G.B. Tiepolo 11, 34131 Trieste, Italy \\ ${ }^{4}$ INAF Osservatorio Astronomico di Trieste, via G.B. Tiepolo 11, 34131 Trieste, Italy \\ 5 Zentrum für Astronomie der Universität Heidelberg, Landessternwarte, König stuhl 12, 69117 Heidelberg, Germany \\ ${ }^{6}$ GEPI, Observatoire de Paris, CNRS, Université Paris Diderot, Place Jules Janssen, 92190 Meudon, France \\ 7 Université de Picardie Jules Verne, 33 rue Saint Leu, Amiens, France
}

Received 30 September 2011 / Accepted 19 January 2012

\section{ABSTRACT}

Context. Recently, the abundance of $\mathrm{P}$ was measured for the first time in disk stars. This provides the opportunity of comparing the observed abundances with predictions from theoretical models.

Aims. We aim at predicting the chemical evolution of $\mathrm{P}$ in the Milky Way and compare our results with the observed $\mathrm{P}$ abundances in disk stars to derive constraints on the P nucleosynthesis.

Methods. We adopted the two-infall model of galactic chemical evolution, which is a good model for the Milky Way, and computed the evolution of the abundances of $\mathrm{P}$ and $\mathrm{Fe}$. We adopted stellar yields for these elements from different sources. The element $\mathrm{P}$ is expected to form mainly in type-II supernovae, whereas Fe is mainly produced by type-Ia supernovae.

Results. Our results confirm that to reproduce the observed trend of $[\mathrm{P} / \mathrm{Fe}] \mathrm{vs}$. $[\mathrm{Fe} / \mathrm{H}]$ in disk stars, $\mathrm{P}$ must be formed mainly in massive stars. However, none of the available yields for $\mathrm{P}$ can reproduce the solar abundance of this element. In other words, to reproduce the data one needs to assume that massive stars produce three times more $\mathrm{P}$ than predicted.

Conclusions. We conclude that the entire available yields of $\mathrm{P}$ from massive stars are largely underestimated and that nucleosynthesis calculations should be revised. We also predict the $[\mathrm{P} / \mathrm{Fe}]$ expected in halo stars.

Key words. Galaxy: abundances - Galaxy: evolution

\section{Introduction}

Recently, Caffau et al. (2011) have measured the P abundance in a sample of $20 \mathrm{cool}$ stars in the Galactic disk. They found that the $[\mathrm{P} / \mathrm{Fe}]$ ratio behaves like the $[\mathrm{S} / \mathrm{Fe}]$ ratio, namely that it increases toward lower metallicity stars. This was the first time that $\mathrm{P}$ was observed in Galactic stars even though $\mathrm{P}$ is among the top 20 most abundant elements in the Universe. There is only one single stable isotope of $\mathrm{P},{ }^{31} \mathrm{P}$, which is thought to be formed by neutron capture on ${ }^{29} \mathrm{Si}$ and ${ }^{30} \mathrm{Si}$ in massive stars. By means of a detailed model for the chemical evolution of the Milky Way one can compute the $\mathrm{P}$ evolution and compare it to the observations. This can allow us to understand the origin of this element and impose constraints on its formation inside stars. In this paper, we adopt a good model for the chemical evolution on the Galaxy that was already tested on many chemical species (François et al. 2004). In particular, the model includes stellar nucleosynthesis and supernova progenitors ( $\mathrm{SNe} \mathrm{Ia}, \mathrm{II}, \mathrm{Ib} / \mathrm{c}$ ) as well as the stellar lifetimes. The stellar yields of $\mathrm{P}$ have been computed by several authors such as Woosley \& Weaver (1995) and Kobayashi et al. (2006) as functions of the initial stellar metallicity. In this paper we compare the predictions of our chemical evolution model for $\mathrm{P}$, obtained by including different stellar yields, with the recent data. In Sect. 2 we briefly describe the chemical evolution model that we adopted, in Sect. 3 we describe the observational data.
In Sect. 4 the results are compared to the data and we draw our conclusions in Sect. 5.

\section{The chemical evolution model}

The role played by SNe of different types in the chemical evolution of the Galaxy has been computed by several authors in the past years (Matteucci \& Greggio 1986; Matteucci \& François 1989; Yoshii et al. 1996; Chiappini et al. 1997, 2001; Kobayashi et al. 2006; Boissier \& Prantzos 1999; François et al. 2004 among others). Here we refer to the model of Chiappini et al. (1997), the so-called two-infall model for the evolution of the Milky Way. A thorough description of this model can be found in Chiappini et al. $(1997,2001)$ and François et al. (2004) and we refer the reader to these papers for details. In this model it is assumed that the stellar halo formed on a relatively short timescale (1-2 Gyr) by means of a first infall episode, whereas the disk formed much more slowly, mainly out of extragalactic gas, through a second infall episode. The timescale for the disk formation is assumed to increase with the galactocentric distance ( $\tau=7 \mathrm{Gyr}$ at the solar circle), thus producing an "inside-out" scenario for the disk formation. The Galactic disk is divided into several rings, $2 \mathrm{kpc}$ wide, without exchange of matter between them. The model can follow the evolution of several chemical elements in detail, including $\mathrm{H}, \mathrm{D}, \mathrm{He}, \mathrm{C}, \mathrm{N}, \mathrm{O}, \alpha$-elements, 
Fe and Fe-peak elements, s- and r-process elements. The star formation rate (SFR) adopted for the Milky Way is a function of both surface gas density and total surface mass density. This SFR is proportional to a power $k=1.5$ of the surface gas density and to a power $h=0.5$ of the total surface mass density. In the Milky Way model we also assumed a surface density threshold below which the SFR stops, according to Kennicutt (1989). As a consequence of this, the star formation rate goes to zero every time that the gas density decreases below the threshold $\left(\sim 7 M_{\odot} \mathrm{pc}^{-2}\right)$. The efficiency of SF is $v=1 \mathrm{Gyr}^{-1}$ during the disk and $2 \mathrm{Gyr}^{-1}$ in the halo phase. This model reproduces the majority of the features of the solar vicinity and the whole disk.

\subsection{Nucleosynthesis and stellar evolution prescriptions}

We adopted the yields for $\mathrm{P}$ and $\mathrm{Fe}$ originating in massive stars from Woosley \& Weaver (1995, hereafter WW95) and Kobayashi et al. (2006, hereafter K06) as functions of stellar metallicity. The yields for the same elements originating from SNe Ia are from Iwamoto et al. (1999), their model W7. In particular, each SN Ia is assumed to eject the same mass, the Chandrasekhar mass $\left(\sim 1.4 M_{\odot}\right)$; of this mass $\sim 0.6 M_{\odot}$ is in the form of $\mathrm{Fe}$ and $\sim 3.57 \times 10^{-4} M_{\odot}$ is in the form of P. It is therefore clear that $\mathrm{SNe}$ Ia are negligible producers of P. On the other hand, ${ }^{31} \mathrm{P}$ should be produced during $\mathrm{O}$ - and $\mathrm{Ne}$ - shell burnings in massive stars, although a large part of ${ }^{31} \mathrm{P}$ can be destroyed by $(\mathrm{p}, \alpha)$ reactions to become ${ }^{28} \mathrm{Si}$.

\section{The observational data}

The sample of observed stars is formed by $20 \mathrm{G}-\mathrm{F}$ bright dwarfs $\left(3.3 \leq J_{\text {mag }}<7\right)$, with stellar parameters in the ranges: $5765 \leq$ $T_{\text {eff }} \leq 6470 \mathrm{~K}, 3.90 \leq \log g<4.5,-0.91 \leq[\mathrm{Fe} / \mathrm{H}] \leq 0.28$.

The phosphorus abundance was derived from CRIRES spectra observed at the VLT-Antu $8 \mathrm{~m}$ telescope in service mode during ESO period 86. The setting was centered at $1059.6 \mathrm{~nm}$ in order 54. Four P lines of Mult. 1 were visible in detectors 2 and 3. The spectral resolution was $R=100000$, the signal-to-noise ratios achieved were in the range 50-400. For the Adaptive Optics correction, computed on axis, the target star itself was used.

\section{Results for phosphorous}

We ran several models including different prescriptions for the $\mathrm{P}$ and Fe yields from massive stars. In some of the models we mixed yields from different sources, which is allowed because yields of some specific elements, in particular those of $\mathrm{P}$ and $\mathrm{Fe}$, are still uncertain, because of the different input physics adopted by different authors. As a consequence, we can derive constraints on the nucleosynthesis calculations by means of our chemical evolution models.

The models are:

- Model 1: with the P yields from massive stars of K06 and the Fe yields from massive stars of WW95 considering only the solar chemical composition. This choice was made because these Fe yields reproduce the $[\mathrm{X} / \mathrm{Fe}]$ vs. $[\mathrm{Fe} / \mathrm{H}]$ relations better (see François et al. 2004);

- Model 2: with the P yields from massive stars of K06 and metallicity dependent $\mathrm{Fe}$ yields from massive stars of WW95;

- Model 3: with the metallicity-dependent P yields from massive stars of WW95 and the Fe yields from massive stars of WW95 considering only the solar chemical composition;

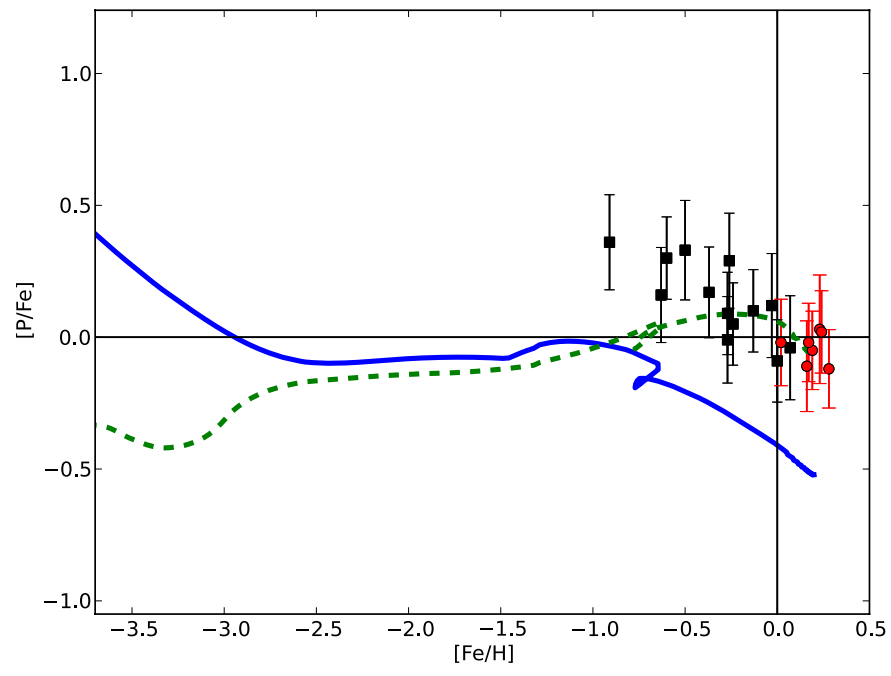

Fig. 1. Predictions from Model 1 (blue solid line) and Model 3 (green dashed line). The $\mathrm{P}$ and $\mathrm{Fe}$ abundances in both models are normalized with the absolute solar values of Caffau et al. (2011). In Table 1 we list the predicted ratios at the age of formation of the solar system. Data from Caffau et al. (2011): the circles indicate stars with planets.

- Model 4: with metallicity-dependent P and Fe yields from massive stars of WW95;

- Model 5: with P and Fe metallicity-dependent yields from massive stars of $\mathrm{K} 06$;

- Model 6: where the yields of P and Fe from massive stars are from K06 and include also hypernovae (hypernovae are stars with $M>20 M_{\odot}$ and explosion energies higher than normal $\mathrm{SNe}$ II);

- Model 7: the only difference compared to model 5 is that in this model the $\mathrm{P}$ yields in massive stars are artificially increased by a factor of $\sim 3$;

- Model 8: the only difference compared to model 6 is that in this model the $\mathrm{P}$ yields in massive stars are artificially increased by a factor of $\sim 2.75$.

To obtain the $[\mathrm{P} / \mathrm{Fe}]$ ratios, we normalized our model results for $\mathrm{P}$ and $\mathrm{Fe}$ with the observed absolute solar abundances obtained by Caffau et al. (2011). In this way, one can see at the same time whether the model predictions fit the trend and also the solar abundances. The predicted solar abundances are the abundances that the model predicts for the ISM 4.5 Gyr ago, the time of formation of the solar system.

In Table 1 we show a summary of the nucleosynthesis prescriptions used for $\mathrm{P}$ and $\mathrm{Fe}$ in the models and the difference between the predicted solar abundances and the observed ones by Caffau et al. (2011). Note that the yields of $\mathrm{P}$ and Fe from $\mathrm{SNe}$ Ia are from Iwamoto et al. (1999) in all models.

In Fig. 1 we show the results of Models 1 and 3. As one can see, Model 1 agrees well with the observed trend in the Galactic disk, although the curve is shifted to lower values than observed. This is because while the solar Fe abundance predicted by this model agrees well with the observed one, the P solar abundance is too low. In particular, to reproduce the P solar abundance we need to artificially increase the yields of $\mathrm{P}$ from massive stars by a factor of $\sim 3$. On the other hand, we note that the results of Model 3 are not able to reproduce the trend of the $[\mathrm{P} / \mathrm{Fe}]$ ratio in the Galactic disk, although the predicted solar value is almost acceptable $(+0.06$ dex, in Table 1$)$.

In Fig. 2 we show Models 2 and 4: Model 2 agrees well with the observed trend in the Galactic disk, whereas for Model 4 
Table 1. Nucleosynthesis prescriptions for the various models.

\begin{tabular}{lccc}
\hline \hline Model & P from massive stars & Fe from massive stars & {$[\mathrm{P} / \mathrm{Fe}]_{\odot}$ model } \\
\hline 1 & K06 & WW95 solar chem. comp. & -0.41 \\
2 & K06 & WW95 met.dep. & -0.42 \\
3 & WW95 met. dep. & WW95 solar chem comp. & +0.06 \\
4 & WW95 met. dep. & WW95 met.dep. & +0.05 \\
5 & K06 & K06 & -0.40 \\
6 & K06+ Hypern. & K06+ Hypern & -0.37 \\
7 & $3 \times$ K06 & K06 & 0.00 \\
8 & $2.75 \times$ K06 + Hypern. & K06+ Hypern. & 0.00 \\
\hline
\end{tabular}

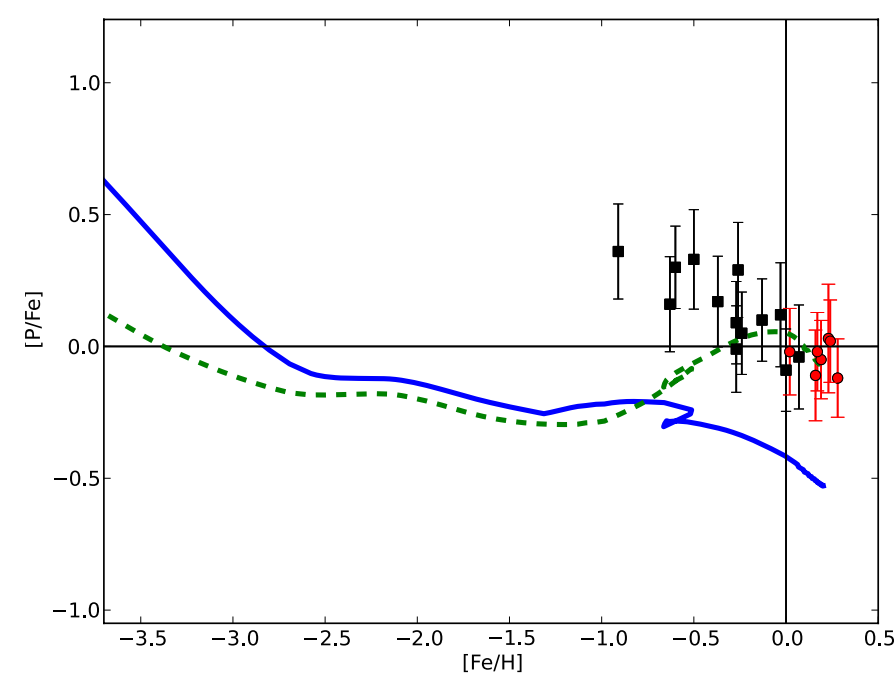

Fig. 2. Predictions from Model 2 (blue solid line) and Model 4 (green dashed line). The $\mathrm{P}$ and $\mathrm{Fe}$ abundances in both models are normalized with the absolute solar values of Caffau et al. (2011). In Table 1 we list the predicted ratios at the age of formation of the solar system. The data are the same as in Fig. 1.

the trend is not reproduced, although, as for Model 3, the predicted solar value could be acceptable $(+0.05$, Table 1$)$. To obtain a good agreement with the observed solar abundance of $\mathrm{P}$ for Model 2, we again need to artificially increase the yields of $\mathrm{P}$ by a factor of $\sim 3$.

In Fig. 3 we show the predictions of Model 5 with metallicity-dependent yields from K06 for both P and Fe. Even for these yields we predict a solar ratio of $[\mathrm{P} / \mathrm{Fe}]_{\odot}$ too low by about -0.40 dex. In other words, although the trend of $[\mathrm{P} / \mathrm{Fe}]$ in the Galactic disk is well reproduced, the whole curve should be shifted upward by 0.4 dex to gain a good agreement with the data.

In Fig. 3 we also show the predictions of Model 6 with the metallicity-dependent yields from K06 for both P and Fe, but for stars more massive than $20 M_{\odot}$ we adopted the yields for hypernovae, instead of those for normal SNe II. The agreement with the observed solar ratio here is slightly better, $[\mathrm{P} / \mathrm{Fe}]_{\odot} \sim$ -0.37 dex, although the problem of the insufficiently predicted solar P abundance remains.

The agreement of Models 1, 2, 5, and 6 with the trend of the data and the disagreement with the observed solar abundances suggests that the major producers of $\mathrm{P}$ in the Universe are probably core-collapse supernovae and that the theoretical yields from these supernovae are underestimated by a factor of $\sim 3$. However, the results of Model 6 with yields from hypernovae differ substantially from those obtained with normal yields from $\mathrm{SNe}$ II at low metallicities. Indeed, the model with

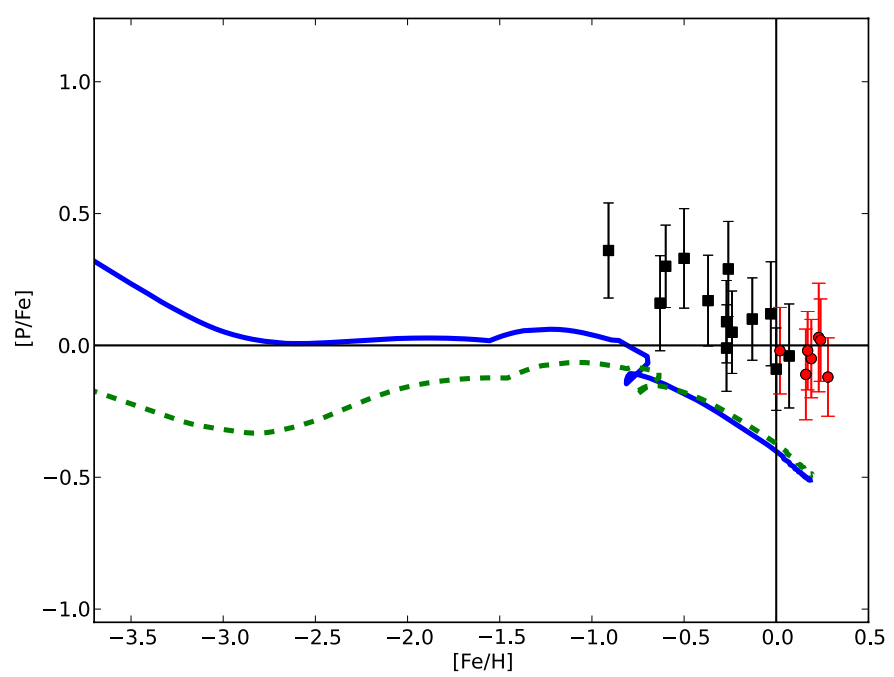

Fig. 3. Predictions from Model 5 (blue solid line) and Model 6 (green dashed line). The $\mathrm{P}$ and $\mathrm{Fe}$ abundances in both models are normalized with the absolute solar values of Caffau et al. (2011). In Table 1 we list the predicted ratios at the age of formation of the solar system. The data are the same as in Fig. 1.

normal SNII yields predicts an overabundance of $\mathrm{P}$ relative to $\mathrm{Fe}$ at low metallicity of $\sim+0.5$ dex, whereas the model with hypernova yields predicts a lower $[\mathrm{P} / \mathrm{Fe}]$ ratio, namely an overabundance of $\sim+0.2-0.3$ dex. Clearly, these predictions await to be proven by future data in low-metallicity halo stars.

On the other hand, the trend of abundances of $[\mathrm{P} / \mathrm{Fe}]$ in the Galactic disk as predicted by Models 3 and 4, adopting the metallicity-dependent yields of $\mathrm{P}$ and Fe from massive stars by WW95, disagrees with observations even if we normalize the model results to their predicted solar abundances. The predictions of Model 4 are very similar to those of Timmes \& al. (1995): these authors adopted the same yields from massive stars for $\mathrm{P}$ and $\mathrm{Fe}$ as in our Model 4, and showed the predicted $[\mathrm{P} / \mathrm{Fe}]$ vs. $[\mathrm{Fe} / \mathrm{H}]$, but no data for $\mathrm{P}$ were available at that time.

We can also infer from our numerical simulations that the theoretical predictions from different authors for $\mathrm{Fe}$ are similar, whereas for P the yields by K06 and by WW95 are significantly different. We therefore conclude that the yields of P by K06 are more accurate than those of WW95, since the former can well reproduce the trend of $[\mathrm{P} / \mathrm{Fe}]$ vs. $[\mathrm{Fe} / \mathrm{H}]$ and the $\mathrm{P}$ solar abundance by increasing them by a factor of $\sim 3$.

For this reason and for the sake of using a more homogeneous set of yields, we only show the results obtained by including the K06 yields either with or without hypernovae in the next figure. In particular, Model 7 is based on normal massive star yields, whereas Model 8 is based on hypernovae yields. In these models, shown in Fig. 4, we applied the necessary factor in the 


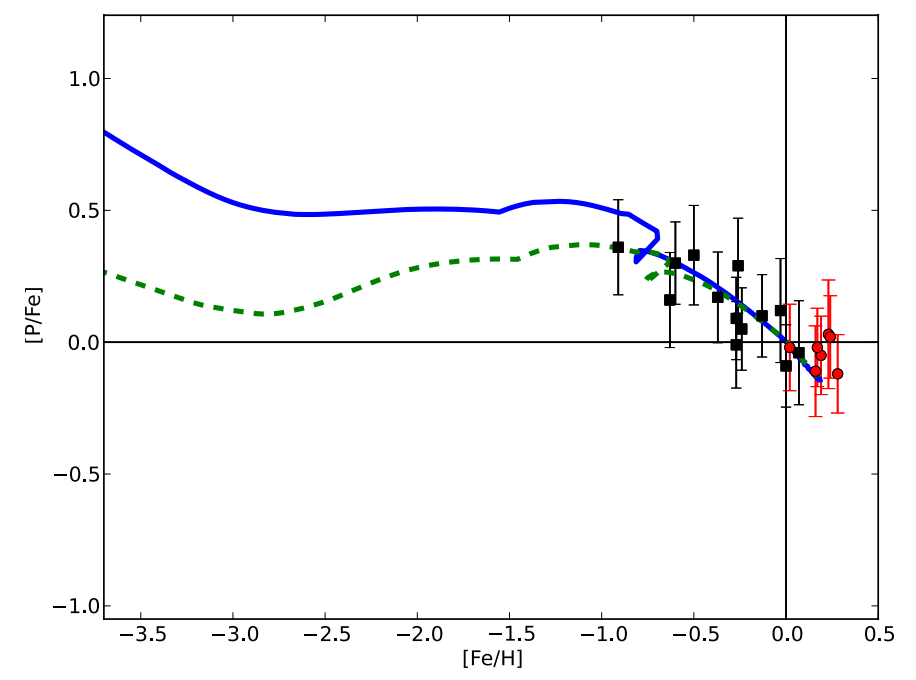

Fig. 4. Predictions for $[\mathrm{P} / \mathrm{Fe}]$ from Model 7 (blue solid line) and Model 8 (green dashed line). The $\mathrm{P}$ and $\mathrm{Fe}$ abundances in both models are normalized with the absolute solar values of Caffau et al. (2011). Data as in Fig. 1.

production of $\mathrm{P}$ in massive stars to reproduce the observed solar abundance of P. Indeed Fig. 4 shows that the predicted curve passes exactly by zero and this is because our model can reproduce the solar abundance of $\mathrm{Fe}$ and that of $\mathrm{P}$ at the same time. It is worth noting that adopting the hypernova yields, which are higher for $\mathrm{P}$ than those for normal core-collapse $\mathrm{SNe}$, the yields need to be increased by a factor of $\sim 2.75$, instead of $\sim 3$. With the corrected yields and the observed solar abundance, the predicted $[\mathrm{P} / \mathrm{Fe}]$ for $[\mathrm{Fe} / \mathrm{H}]<-1.0$ dex is on average $+0.2-0.3$ dex.

\section{Conclusions}

We compared model predictions by adopting different sets of yields with recent data on the abundance of $\mathrm{P}$ in the Galactic disk. Our conclusions can be summarized as follows:

- The observed decrease of the abundance of $[\mathrm{P} / \mathrm{Fe}]$ in Galactic disk stars suggests that $\mathrm{P}$ is mainly produced by core-collapse $\mathrm{SNe}$ with a small contribution from $\mathrm{SNe}$ Ia. Otherwise, if $\mathrm{SNe}$ Ia were important, the $[\mathrm{P} / \mathrm{Fe}]$ ratio would remain fairly constant.

- The metallicity-dependent yields of P from massive stars of K06 together with the P yields from SNe Ia from Iwamoto et al. (1999) adequately reproduce the data if the yields from massive stars are increased by a factor of $\sim 3$. This suggests that the yields of $\mathrm{P}$ available in the literature are underestimated. Both the neutron-rich isotopes of ${ }^{29,30} \mathrm{Si}$ and ${ }^{31} \mathrm{P}$, which derive from neutron capture on the two $\mathrm{Si}$ isotopes, are produced in the oxygen- and neon-burning shells in massive stars (WW95). Therefore, to obtain a higher P production, the $\mathrm{O}$ and $\mathrm{Ne}$ shell- burnings and the neutron capture on the ${ }^{29,30} \mathrm{Si}$ isotopes and the destruction of ${ }^{31} \mathrm{P}$ by $(\mathrm{p}, \alpha)$ reactions should be revised.

- We also predict the behavior of $[\mathrm{P} / \mathrm{Fe}]$ in halo stars and suggest that it will likely show a plateau between $[\mathrm{Fe} / \mathrm{H}]=$ -1.0 and -3.0 dex corresponding to $[\mathrm{P} / \mathrm{Fe}] \sim+0.5 \operatorname{dex}$ if yields (corrected) from normal $\mathrm{SNe}$ II are adopted, and to $\sim+0.2$ dex if hypernova yields (corrected) are adopted. To distinguish between these two cases, we await observations of the $\mathrm{P}$ abundance in halo stars.

Acknowledgements. GC thanks the Department of Mathematics and Computer Science of the University of Udine for its hospitality.

\section{References}

Boissier, S., \& Prantzos, N. 1999, MNRAS, 307, 857

Caffau, E., Bonifacio, P., Faraggiana, R., \& Steffen, M. 2011a, A\&A, 532, 98

Caffau, E., Ludwig, H.-G., Steffen, M., Freytag, B., \& Bonifacio, P. 2011b, SoPh, 268,255

Chiappini, C., Matteucci, F., \& Gratton, R. 1997, ApJ, 477, 765

Chiappini, C., Matteucci, F., \& Romano, D. 2001, ApJ, 554, 1044

François, P., Matteucci, F., Cayrel et al. 2004, A\&A, 421, 613

Iwamoto, K., Brachwitz, F., Nomoto, K., et al. 1999, ApJS, 125, 439

Kennicutt, R. C. Jr, 1989, ApJ, 344, 685

Kobayashi, C., Umeda, H., Nomoto, K, Tominaga, N., \& Ohkubo, T. 2006, ApJ, 653, 1145 (K06)

Matteucci, F., \& François, P. 1989, MNRAS, 239, 885

Matteucci, F., \& Greggio, L. 1986, A\&A, 154, 279

Timmes, F. X., Woosley, S. E., \& Weaver, T. A. 1995, ApJS, 98, 617

Yoshii, Y., Tsujimoto, T., \& Nomoto, K. 1996, ApJ, 462, 266

Woosley, S. E., \& Weaver, T. A. 1995, ApJS, 101, 181 (WW95) 